\title{
Long-Term Follow-Up of Orally Administered Diacetylmorphine Substitution Treatment
}

\author{
Ulrich Frick $^{\mathrm{a}-\mathrm{c}}$ Jürgen Rehm ${ }^{\mathrm{a}} \mathrm{d-f} \quad$ Daniele Zullino $^{\mathrm{g}}$ Manrique Fernando $^{\mathrm{h}}$ \\ Gerhard Wiesbeck $^{i}$ Jeannine Ammann ${ }^{a}$ Ambros Uchtenhagen ${ }^{a}$ \\ ${ }^{a}$ Research Institute for Public Health and Addiction, Zurich, Switzerland; ${ }^{b}$ Carinthia University of Applied Sciences, \\ Department of Healthcare Management, Feldkirchen, Austria; 'Psychiatric University Hospital, Regensburg,

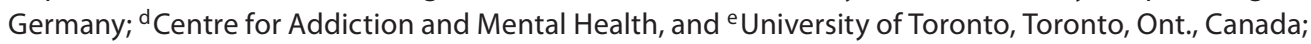 \\ ${ }^{f}$ Epidemiological Research Unit, Technische Universität Dresden, Klinische Psychologie und Psychotherapie,

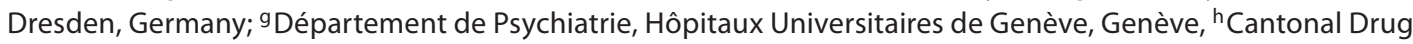 \\ Counselling, Reinach, and 'Division of Substance Use Disorders, Department of Psychiatry, University of Basel, \\ Basel, Switzerland
}

\section{Key Words}

Opioid dependence $\cdot$ Heroin substitution treatment $\cdot$ Oral application · Open-label study · Prospective cohort • Bias servation, and 0.005 events per application year in the other group. Conclusions: Because of their feasibility and safety over years, DAM tablets may be a valuable long-term therapeutic alternative.

Copyright $\odot 2010$ S. Karger AG, Basel

\begin{abstract}
Background: To assess the long-term course of the feasibility and safety of orally administered heroin [diacetylmorphine (DAM)] tablets in substitution treatment of severely addicted opioid users. Design: Open-label, prospective cohort study with 2 non-randomly assigned treatment arms: DAM tablets only $(n=128)$ or DAM tablets combined with injected DAM and/or other opioids $(n=237)$. The average duration of the observation period was 62 months. Study endpoints were the time to discharge from treatment and the number of serious adverse events. Results: Both patient groups had a higher than $70 \%$ retention rate after the first 48 months of treatment, with similar long-term retention rates (after 8 years both groups had retention over $50 \%$ ). The physician-verified rate of serious adverse events was 0.01 events per application year among the exclusively oral substitution group (intention-to-treat analysis) during the last year of ob-
\end{abstract}

\section{Introduction}

Maintenance treatment, especially with methadone, has become the standard treatment for opioid dependence in numerous countries and has been recommended by international organizations [1-4]. Not all opioiddependent patients, however, fully respond to methadone maintenance treatment [5]. The medical prescription of intravenous diacetylmorphine (DAM) to chronic treatment refractory heroin-dependent patients has been considered as a second-line therapy in several countries and its feasibility and effectiveness has been confirmed in cohort studies [6,7] and controlled trials [8-10]. Patients who had never or not regularly injected had to be excluded from intravenous heroin substitution for ethical con-

\section{KARGER \\ Fax +41613061234 E-Mail karger@karger.ch} www.karger.com
() 2010 S. Karger AG, Basel $1022-6877 / 10 / 0163-0131 \$ 26.00 / 0$

Accessible online at:

www.karger.com/ear
Ulrich Frick

Carinthia University of Applied Sciences

Hauptplatz 12

AT-9560 Feldkirchen in Kärnten (Austria)

Tel. +43 590500 4116, E-Mail u.frick@fh-kaernten.at 
siderations as they cannot be introduced to an application form which bears more health risks. The reasons for the oral use of illegal opioids vary and include cultural influences, fear of risks of infection and the inability to find veins for injection. Consequently, alternatives in HAMT were explored by studying inhalable heroin in the Netherlands [11], morphine tablets [12], and intramuscular (i.m.) and oral application (p.o.) modes in Switzerland $[13,14]$. The latter options were explored because inhalable heroin was not possible in Switzerland or many other countries for legal reasons. Among other alternatives, the combination of immediate-release and slow-release tablets of DAM [15], as introduced within the Swiss HAMT, provided the most promising results with respect to retention rates and serious adverse events [16]. Both galenic forms were developed by the Swiss Federal Office of Public Health in 1998 for use in heroin-assisted therapy and each contains $200 \mathrm{mg}$ of diamorphine hydrochloride.

Gyr et al. [17] compared high-dose intravenous (200$690 \mathrm{mg}$ ) and oral (capsules, controlled-release tablets) DAM preparations, and found DAM to be detectable in plasma only after intravenous administration. Oral doses and dosage intervals were described to be sufficient to produce flash and high effects among participants. In a further study, oral DAM $(<600 \mathrm{mg}$ ) was well absorbed in the intestine, but underwent excessive first-pass deacetylation to morphine with a rather high mean morphine bioavailability of $64-72 \%$, but resulted in negligible systemic DAM and monoacetylmorphine exposures [14]. Oral DAM was absorbed more rapidly and to a greater extent than a concomitant test dose of morphine-d3 [14]. Confirming the preliminary results on DAM, Halbsguth et al. [18] demonstrated that morphine bioavailability depends on:

(1) The particular drug administered (morphine absorption from oral DAM was found to be faster than from oral morphine, leading to higher $\mathrm{C}_{\max }$ and shorter $\mathrm{t}_{\max }$ values observed in both the investigated group of opioiddependent and opioid-naïve subjects, $\mathrm{n}=8$ per group).

(2) Prior opioid exposure (for the systemic morphine exposure achieved from oral DAM a doubling of the maximum morphine concentration at about half the maximum time were observed in opioid-dependent compared with opioid-naïve subjects).

(3) Dose (a dose effect was observed for oral DAM, increasing morphine bioavailability up to $64 \%$ at the highest dose of $598 \mathrm{mg}$; moreover, morphine bioavailability increased with co-administration of DAM).
The study [18] presents pharmacokinetic advantages of oral DAM over oral morphine, resulting in a compliance-relevant faster onset of drug and more efficient bioavailability for substitution treatment. Thus, morphine from oral DAM was more rapidly and more completely absorbed than from oral morphine. Therefore, oral DAM could be seen as an active pro-drug of morphine reaching a higher level of systemic morphine faster than from morphine itself [14].

As previously mentioned [16], the results of an intention-to-treat analysis of the current open-label prospective cohort study for DAM tablets within the Swiss HAMT after an observation period of 1 year have been reported. DAM p.o. treatment was part of the overall Swiss HAMT that addressed the treatment needs of patients ineligible for DAM i.v. or who had or wanted to switch to DAM p.o. (see below for details). In the Frick et al. study [16], the retention rates after 1 year in DAM tablets-only group (0.804, 95\% CI: $0.735-0.873)$ and in the subgroup combining oral application of DAM with intravenous application or other opioids $(0.843$, 95\% CI: $0.797-$ 0.889 ) were higher than in the historical controls, i.e. the Swiss cohort of patients that had been intravenously substituted with DAM (1-year retention rate $=0.70)$. In addition, rates of serious adverse events under study medication (tablets only $=0.038$ per application year; tablets in combination $=0.028$ per application year) were comparable to the historical rate of the Swiss heroin-assisted treatment (0.043).

The present analyses report the results for the feasibility and safety of oral DAM using the same study endpoints with prolonged observation period: for feasibility, the period between a patient's individual start date of oral medication and July 2008 was evaluated; for safety, the first 2 years after the start of the formal drug approval study were used.

\section{Methods}

The study design was an open-label prospective cohort study with 2 non-randomly allocated treatment arms to be compared. Additionally, the pooled cohort of both treatment arms is compared in its long-term outcome (retention from first tablet to July $28,2008)$ to the complete population of injecting DAM users in the Swiss HAMT (from individual treatment entry to the same time point).

Eligibility

Patients who were already admitted to the Swiss heroin-assisted treatment program (for general eligibility criteria for this treatment program for severely addicted opioid users, see [19]) were
Frick/Rehm/Zullino/Fernando/ Wiesbeck/Ammann/Uchtenhagen 


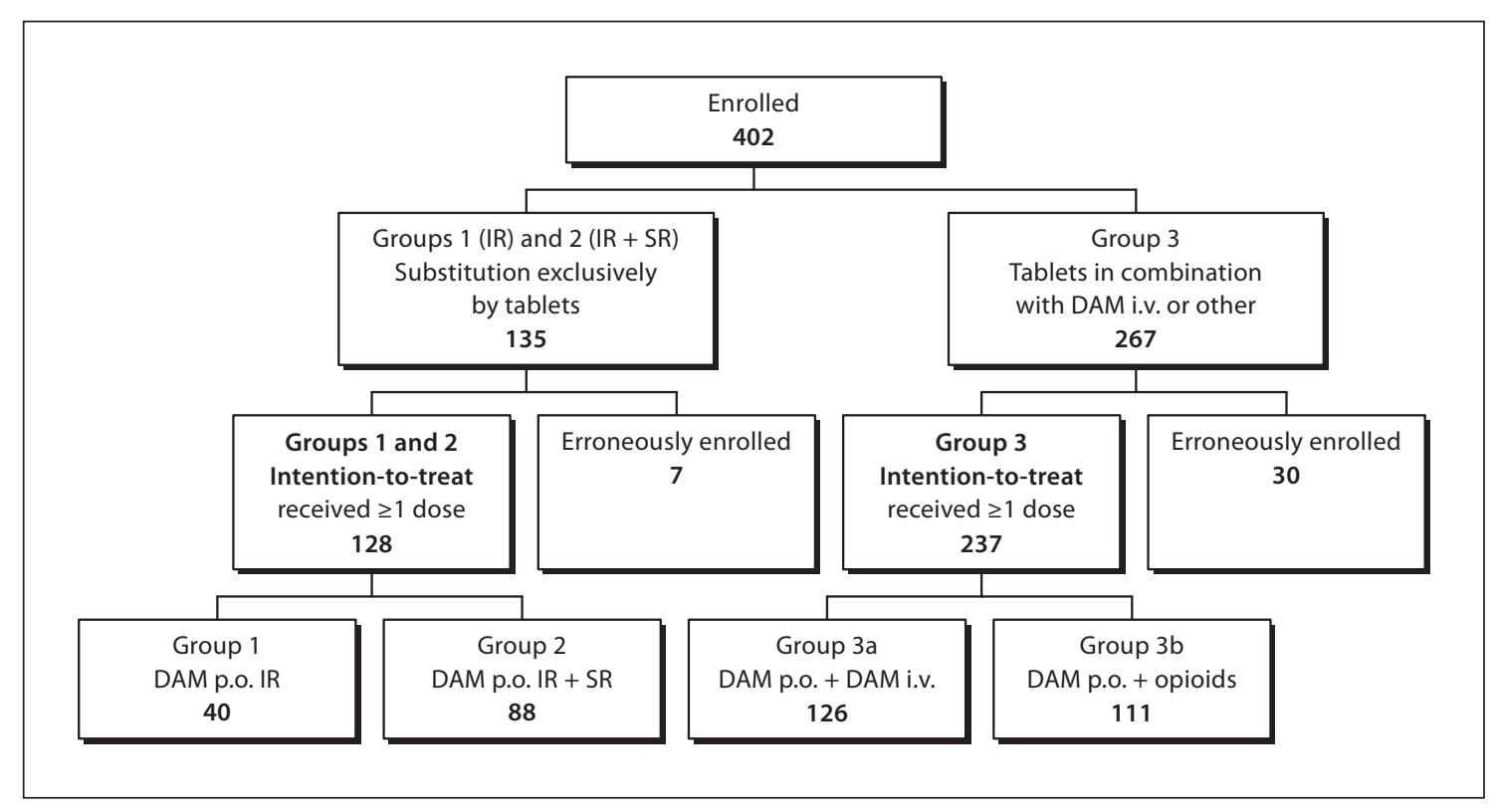

Fig. 1. Study population. IR = Immediate release; $S R=$ slow release.

eligible to be enrolled into this study if one or more of the following conditions were fulfilled: (1) the status of their veins prohibited further intravenous application of DAM, (2) they had used opioids prior to the substitution program exclusively via inhalation or sniffing, and intravenous use, therefore, meant an increase in health risk for them, or (3) they wished to reduce their health risk gradually or abruptly, but in any case permanently shifting from intravenous to the oral application of DAM.

All 402 patients already receiving DAM tablets in the Swiss heroin-assisted treatment, or scheduled to receive such tablets from the reference date of each treatment center, were enrolled in the study (fig. 1). Twenty-one of the 23 existing Swiss outpatient treatment centers recruited patients. For the remaining 2 centers, there were no patients fulfilling the inclusion criteria.

\section{Treatment Allocation and Study Groups}

All patients were treated according to Swiss heroin-assisted treatment protocol, as specified by and under permission of the Federal Office of Public Health on a patient-named basis. Treatment included psychosocial services at least once per month, and somatic interventions if necessary. Opiate dosage and number of administrations were prescribed according to the patient's needs. The treatment centers were open 3 times per day, 7 days per week. In groups 1 and 2, no other substitution medication was given (for protocol violations, see below). Consumption was monitored by a self-reported questionnaire and urinalysis.

Groups 1 and 2 (Tablets Only). A total of 128 patients received a medication regimen providing DAM exclusively in tablet form [either immediate-release tablets alone (group 1) or combining immediate release and slow-release tablets (group 2)].

Group 3 (Tablets and Other Opioids). A combination of DAM tablets with either injectable DAM or other noninjectable opioids, like morphine (as a tablet), was given ( $\geq 1$ dose) to 237 patients (group 3). These patients received intravenous applications only if there was clear evidence of intravenous use prior to the study. With regard to medications other than diamorphine, study group 3 was designed to be a 'left over' category, comprising all combinations of substitution-medication including combinations with methadone or morphine tablets.

At baseline, $26.5 \%$ of all patients in groups 1 and 2, but only $9.7 \%$ of group 3 , had never practiced intravenous application of heroin or other opioids ( $\chi^{2}$ test; $\left.\mathrm{p}<0.001\right)$. With respect to variables predictive for treatment outcome in the cohort entering the Swiss HAMT program between January 1, 2001 and February 29, 2004 [20], no differences were found between patients receiving tablets and those receiving only intravenous applications of heroin.

Within the groups described, 25 patients in groups 1 and 2 (tablets only) and 49 patients in group 3 had not received DAM tablets prior to the start of the study. The remaining patients had some experience with DAM tablets as part of the Swiss heroinassisted treatment. Therefore, this article uses the longest available observation period per patient for evaluation, and counts the duration of oral substitution treatment from the very first day that the patient received a DAM tablet.

\section{Study Period}

The feasibility of oral DAM medication was evaluated for each patient's longest possible observation period, irrespective of the time when he/she was included into the formal drug approval study. Thus, the duration of the observation period was counted as the days from each patient's first medication with DAM tablets until July 18, 2008 when the database was frozen. On average, patients in the tablets only groups had an observation period of 2,253 days (SD 627), while patients in group 3 (tablets and other opioids) had 2,237 days (SD 528). Analyses on adverse events were 


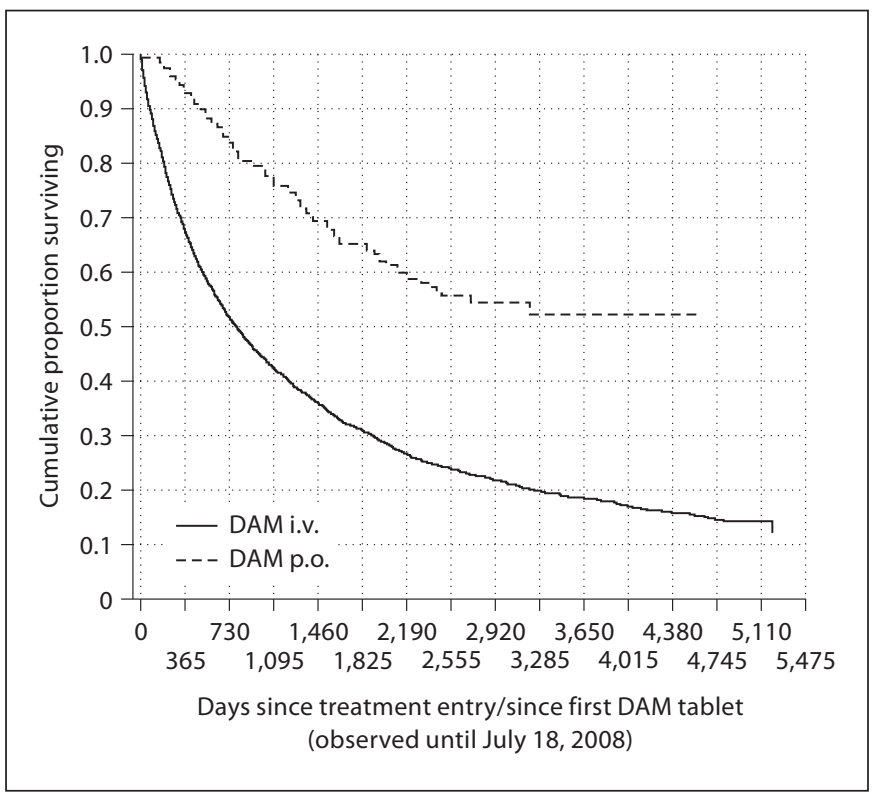

Fig. 2. Retention rate in Swiss heroin-assisted treatment by application type since start (Kaplan-Meier estimator).

performed for the second year after the start of the formal drug approval study (in most cases: January 2005 to January 2006) in order to compare the risk for serious adverse events per application year to the risk calculated for the observation period (November 2003 to December 2004) that was reported by Frick et al. [16].

\section{Study Endpoints}

The main endpoint of the 1-year study by Frick et al. [16] was the retention rate, which was to be compared to the known historical 1-year retention rate of the Swiss heroin substitution program, reported to be a proportion of 0.7 [6]. Sample size calculations were based on this comparison $[16,21]$. Discharge from HAMT was defined as either an explicit ending of treatment by a respective declaration of the patient, formally excluding the patient from the treatment for disciplinary reasons according to the rules of HAMT [19] or by a patient-caused interruption of treatment (i.e. not presenting at the treatment center for longer than 6 weeks without prior information or approval). For this prolonged observation period study, the major study endpoint was defined as the period between the first day a patient received a DAM tablet, and his/her first discharge from HAMT after having received oral medication. When comparing patients with any oral application (pooled groups 1-3) to those exclusively receiving an intravenous application (fig. 2), latter group's day of treatment entry was counted as the starting point.

The safety of orally administered DAM was chosen as the second major study endpoint and was monitored during the study. Data of serious adverse events were collected via the reporting system that has been monitoring the safety of heroin prescription in the Swiss heroin-assisted treatment since 1994. Physicians' reports of adverse events were classified according to the Interna- tional Committee on Harmonization guidelines for clinical trials as grade 3 in case of deaths, life-threatening events, hospitalizations and events causing a permanent noxa or functional disability. A critical value of 4.3 serious adverse events per 100 application years was chosen as a minimum safety standard from previous experience with the intravenous application of DAM [16].

\section{Statistical Analysis}

Time to discharge from HAMT as a major study endpoint displayed censored observations at the day, when the database was frozen (July 18, 2008). Therefore, survival curves were compared by calculating the Kaplan-Meier estimates for the survival function and a log-rank test. SAS Proc Lifetest was used for calculations.

All statistical analyses were performed using intention-totreat principles and therefore included patients with protocol violations ( $\mathrm{n}=24$ in tablets only group) in the originally assigned groups. Patient treatment schemes remained unchanged for the subsequent years of observation (until July 2008).

\section{Approval by Research Ethics Board}

The study protocol, including the extension of the original study period, was approved by all 9 cantonal ethics committees that were responsible for the participating 21 treatment centers.

\section{Results}

\section{Sociodemographic and Other Characteristics at Baseline}

The sociodemographic characteristics have been reported in detail in previous publications $[16,21]$ and will only be summarized here. The majority of patients were men $(n=281 ; 77.0 \%)$, with an average age of 37.1 years (SD 6.4). Most of the participants were living in rented apartments $(\mathrm{n}=266 ; 72.9 \%)$ with 9 people being homeless (2.5\%). 106 patients had at least part-time employment in the unprotected employment market (29.0\%).

Patients had used heroin for longer than a decade before administration of oral DAM [16]. They had high somatic and mental disease burden, as evidenced by rates of $65 \%$ for hepatitis C infection, $11 \%$ for HIV infection, $32 \%$ for personality disorders, $32 \%$ for affective disorders and $15 \%$ with two and more additional psychiatric diagnoses in October 2003.

\section{Major Study Endpoints: Treatment Retention}

The main study endpoint of the prospective cohort study was the retention rate. Figure 3 shows the temporal course of this endpoint.

The retention rate 24 months after the individual start date of oral medication for groups 1 and 2 was $80.7 \%$ (95\% CI: 73.6-87.8\%), which was somewhat lower than the rate of group 3 (87.6\%; 95\% CI: 83.2-92.0\%). For both groups, 
however, these retention rates were significantly higher after more than 2 years than the 1-year retention rate in the historical sample of intravenous heroin-assisted treatment (70\%).

At day 1,349 (3.7 years or 45 months after initial tablets medication) survival curves of both conditions intersected at a retention rate of $72.5 \%$ (95\% CI for group 3: 67.0$78.8 \%$ ). Thereafter, groups 1 and 2 displayed a somewhat more favorable retention rate until day 2,472 (after 82 months or 6.8 years). Then both survival curves intersected a second time at a rate of $56.0 \%$ (95\% CI for groups 1 and 2: 44.4-67.7\%). Groups 1 and 2 displayed a very stable treatment retention rate after this period lasting at $56.0 \%$ for more than 12 years (or 150 months) for the longest observed patients. Group 3 displayed a retention rate of 50.3\% (95\% CI: 39.0-61.4\%) after 8.8 years (or 106 months), which remained stable to the last observation after 11.4 years (or 139 months). Both retention rates can be seen as remarkably high given the length of the treatment period. A formal log-rank test to compare the groups was not possible because of the nonproportional hazard rates. The Kolmogorov-Smirnov test for differences between the shapes of both survival curves yielded a value of $\mathrm{p}<0.001$. This indicates potential disadvantages for the tablets only medication regimen during the first 3 years which were outweighed by the advantages during the years $4-6$; there were no meaningful differences in the long run after 6 years.

Figure 2 shows treatment retention for all DAM p.o. groups combined in comparison to DAM i.v. DAM p.o. was associated with longer treatment retention. After 12 years, the respective estimates for treatment retention were: DAM p.o. patients, $52.1 \%$ (95\% CI at day 3,205: 44.6-59.5\%); DAM i.v. patients, $15.7 \%$ (95\% CI at day 4,373: 14.1-17.3\%).

Retention in a maintenance study is often time-dependent, with the highest dropout rate occurring shortly after receiving a new study medication. As the majority of patients in our study had already been using the study medication before the start of the study, this may have resulted in self-selection bias. It may be possible that in the patients who had been using the study medication before, people at higher risk of dropout had already dropped out by the time the study began, and thus, the high retention rate was caused mainly by these long-term heroin tablet users. We tested this potential bias by comparing the two groups, i.e. those who had been on DAM p.o. before the study began and those who started with DAM p.o. on the first day of the study. Though the confidence intervals overlapped over the full study period



Fig. 3. Retention rate (ITT) in groups 1 and 2 combined (tablets only) versus group 3 (tablets and DAM i.v. or other oral opiods) since start of oral medication (Kaplan-Meier estimator).

(fig. 4), a formal log-rank test yielded a p value of 0.0326 , pointing at a somewhat faster dropout of patients set on tablets in October 2003.

\section{Major Study Endpoint: Adverse Events}

The physician-verified rate of serious adverse events was 0.01 events per application year, or $1 \%$ of affected patients among the exclusively oral substitution group (ITT analysis) during the second year of observation (table 1 , which also gives adverse events during first observation year). This rate was lower than during the first observation year. Combined medication strategies were associated with a rate of 0.005 events per application year $(0.5 \%$ of affected patients), again lower than the first observation year. Neither group deviated significantly from the a priori fixed tolerance limit of $4.3 \%$ of affected patients.

The two serious events (death of one patient in India on study leave substituted by methadone; admission to psychiatric hospital for stabilization and cocaine withdrawal) were not causally related to the study medication. After January 2006 (end of second observation year), registration and monitoring of adverse events followed the routine procedures of Swiss HAMT and, thus, were not available for the purpose of this study. 
Fig. 4. Retention rate of patients receiving DAM tablets prior to October 2003 (start of drug approval study) versus the rate since October 2003.

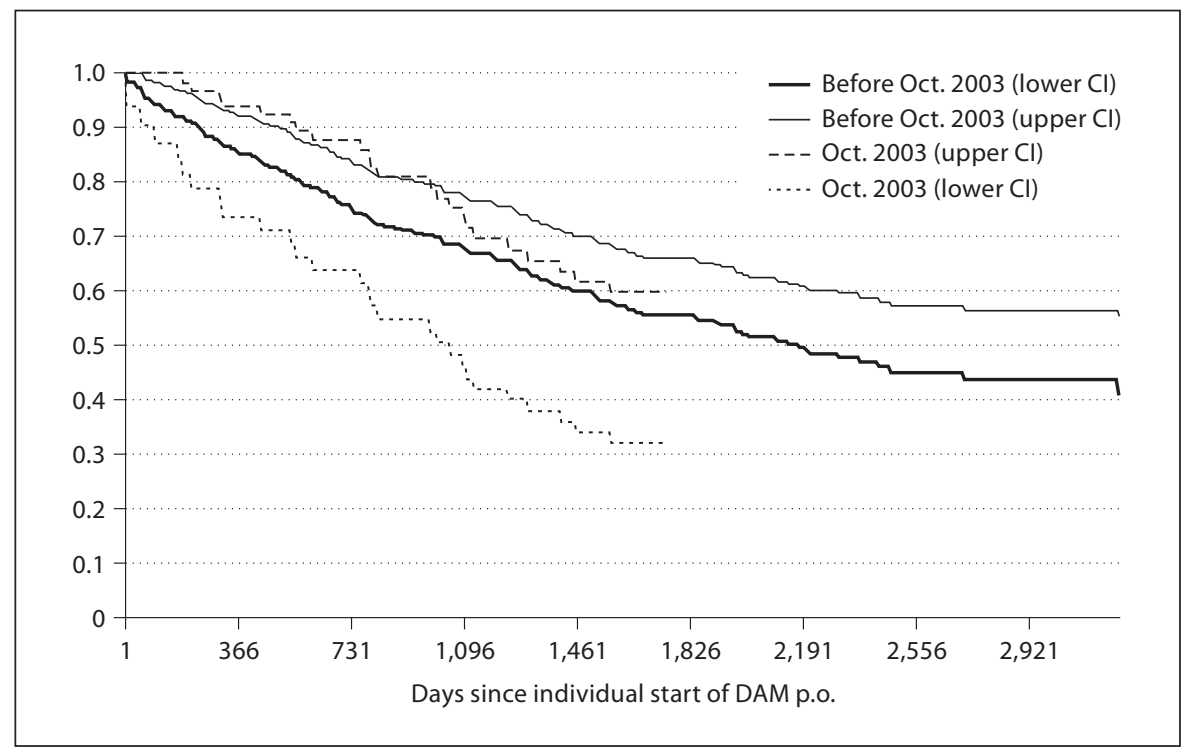

Table 1. Incidence of serious adverse events

\begin{tabular}{|c|c|c|c|}
\hline $\begin{array}{l}\text { Group } 1 \\
\text { (only IR } \\
\text { tablets) }\end{array}$ & $\begin{array}{l}\text { Group } 2 \\
\text { (IR }+ \text { SR } \\
\text { tablets) }\end{array}$ & $\begin{array}{l}\text { Groups } 1 \text { and } 2 \\
\text { combined }\end{array}$ & $\begin{array}{l}\text { Group } 3 \\
\text { (combination of tablets } \\
\text { with opioid medication) }\end{array}$ \\
\hline
\end{tabular}

Observation period 1: November 1, 2003 to January 5, 2005

Events

Patients with event

Patients under risk

Days under risk

Events per year 95\% CI (Poisson) lower limit 95\% CI (Poisson) upper limit

Rate of persons with serious events per year 95\% CI (Poisson) lower limit 95\% CI (Poisson) upper limit

Mean observation time per patient, years

$0 \quad 5$

0

40

15,999

0.000

0.000

0.081

0.000

0.000

0.081

1.10
5

88

32,434

0.056

0.019

0.126

0.056

0.019

0.126

1.00

\section{5}

5

128

48,433

0.038

0.012

0.086

0.038

0.012

0.086

1.03

\section{7}

5

237

90,608

0.028

0.011

0.057

0.020

0.007

0.046 1.05
12

10

365

139,041

0.032

0.016

0.054

0.026

0.013

0.048 1.04

Observation period 2: January 6, 2005 to January 5, 2006

Events

Patients with event

Patients under risk

Days under risk

Events per year 95\% CI (Poisson) lower limit 95\% CI (Poisson) upper limit

Rate of persons with serious events per year 95\% CI (Poisson) lower limit 95\% CI (Poisson) upper limit Mean observation time per patient, years
0

37

12,860

0.000

0.000

0.014

0.000

0.000

0.014

0.95

\section{1}

68

24,121

0.015

0.000

0.052

0.015

0.000

0.051

0.97
1

1

105

36,981

0.010

0.000

0.034

0.010

0.000

0.033

0.96
1

1

203

68,137

0.005

0.000

0.018

0.005

0.000

0.017

0.92
2

2

308

105,118

0.007

0.000

0.019

0.006

0.000

0.017

0.94 


\section{Discussion}

Chronic dependent opioid users are characterized by the persistence of opioid use regardless of the difficulties they experience with their health, the law and in their social environment. DAM tablets offer an effective maintenance treatment for the specific subgroup of refractory and severely addicted opioid users who do not inject. Overall, DAM p.o. substitution has proven to be a safe and feasible treatment for severely opioid-dependent people. Thus, our results may be generalizable to a selection of patients willing to try oral administration. Safety was comparable to DAM i.v., which is already accepted as a regular medication by the Swiss authorities for the treatment of this group. Feasibility, as measured by treatment retention, was even significantly better in DAM p.o. substitution.

There may be alternative settings for nonintravenous DAM substitution. For instance, oral morphine has been suggested as a second-line substitution medication [12], and inhalable DAM has been tested in the Netherlands with success [11]. We hope that these and other alternatives will be tested against DAM p.o. in randomized trials. For inhalable DAM, different forms for inhalation from the Dutch may have to be found to be accepted as medication in some countries, but in principle this is possible. Our hypothesis would be that DAM p.o. would be more effective than other oral opioids including morphine, as the bioavailability is higher and a faster onset can be reached [18]. But these are hypotheses based on basic research; the effects have to be confirmed in randomized trials under real-life conditions.

There were some potential methodological limitations concerning the self-selection process of the participants in our study. A portion of our patients might have chosen oral application of DAM because their attitude towards risks associated with intravenous application had been changing and this change reflected a general process of dissociation from the illegal drug scene. This process could then have determined the low rate of dropouts as well as the low illegal consumption and the observed low rate of serious adverse events. This argument could only be countered by a randomized controlled clinical trial comparing intravenous versus oral application mode of DAM, which would be unethical.

We found hints on a faster dropout process of the 74 patients (18\%) who started with tablets in October 2003. Whether this means the retention rates of the oral DAM groups are positively flawed by omitting potential former dropouts prior to our study cannot be concluded with certainty. It also seems plausible that the historical situation in autumn 2003 was understood by the treating physicians as a 'last chance' to change over the substitution medication of their patients to oral DAM for a long time period, and therefore they recruited in October 2003 a group of patients who were less suited for oral substitution than the previous subgroup.

It may also be argued that the reported retention was only possible because the patients were recruited mainly from patients already in maintenance treatment, thus excluding the high rate of dropouts in the initial treatment period $[6,10]$. Three indirect arguments could be made: opioid addiction is a chronic relapsing disease and, thus, for many opioid-dependent patients, life has been a succession of episodes inside treatments and untreated episodes. Even people entering treatment after 1 year outside treatment have often had multiple treatment episodes before [10, 22], and vice versa: cohorts of originally untreated opioid users show a high prevalence of maintenance treatment episodes after some time [23]. The success of maintenance treatment seems to be relatively independent of prior history. A German trial did not find significant differences in effectiveness of heroin-assisted treatment between people already in methadone treatment or outside the treatment system [10]. Furthermore, in the Swiss study, the success of treatment, as defined by retention rate, was independent of whether it was a first, second or third treatment episode [24]. Therefore, we would hypothesize that the effectiveness of DAM p.o. is independent of whether it is a first treatment episode or if the patient enters this treatment from another treatment. Such a hypothesis must be further examined in new studies.

Finally, the quite 'liberal' definition of retention in the Swiss HAMT is also covering periods of methadone substitution during patients' holidays, for instance. Therefore comparing these retention rates with stricter definitions from outside Switzerland might face a potential 'overestimation' of retention for Swiss patients. But this argument does not apply for comparisons within the Swiss HAMT.

Notwithstanding the methodological limitations mentioned above, the results of our study strongly support the continuation of this treatment for all patients with the mentioned indications for the oral application of HAMT. While other studies may clarify the optimal application of DAM tablets in combination with other opioids or in exclusive prescription, the current practice clearly seems justified by the clinical results. 
Another open question concerns the application of oral DAM as a first-line treatment for refractory opioid users. Currently, intravenous application is the default for everybody, except for users of illegal opioids who do not inject. Given the results above, it should be considered whether the continuation of the status quo of providing a potentially more harmful treatment option (intravenous prescription) as a first-line treatment is justified. Of course, we would need more research with better design and including different endpoints before a truly evidencebased decision to make oral DAM a first-line treatment for refractory opioid users can be made.
Finally, we hope that our results with respect to DAM tablets will lead to a further 'normalization' of the role of HAMT within the continuum of care. Meanwhile diamorphine tables received orphan drug status in Switzerland. In a time when substitution treatment finally has been accepted as the standard of evidence-based opioid treatment $[1,2]$, we see no reason why heroin tablets should be considered any different from other forms of opioid substitution. As already indicated above, further randomized trials using different types of opiates should be conducted to provide the necessary evidence of future guidelines.

\section{References}

1 United Nations Office on Drugs and Crime: Principles of Drug Dependence Treatment. Geneva, WHO, 2008.

2 World Health Organization, United Nations Office on Drugs and Crime, UNAIDS: Substitution Maintenance Therapy in the Management of Opioid Dependence and HIV/ AIDS Prevention: Position Paper. Geneva, WHO, 2004.

3 Gonzalez G, Oliveto A, Kosten TR: Combating opiate dependence: a comparison among the available pharmacological options. Expert Opin Pharmacother 2004;5:713-725.

4 Uchtenhagen A: Substitution management in opioid dependence. J Neural Transm Suppl 2003;66:33-60.

5 Fischer B, Rehm J, Kim G, Kirst M: Eyes wide shut? a conceptual and empirical critique of methadone maintenance treatment (MMT). Eur Addict Res 2005;11:1-9.

6 Rehm J, Gschwend P, Steffen T, Gutzwiller F, Dobler-Mikola A, Uchtenhagen A: Feasibility, safety, and efficacy of injectable heroin prescription for refractory opioid addicts: a follow-up study. Lancet 2001;385:1417-1420.

7 Uchtenhagen A, Gutzwiller F, Dobler-Mikola A, Steffen T, Rihs-Middel M (eds): Prescription of Narcotics for Heroin Addicts Main Results of the Swiss National Cohort Study. Basel, Karger, 1999, p 134.

8 Perneger TV, Giner F, del Rio M, Mino A: Randomised trial of heroin maintenance programme for addicts who fail in conventional drug treatments. BMJ 1998;317:13-18.

9 Oviedo-Joekes E, Brissette S, Marsh DC, Lauzon P, Guh D, Anis A, Schechter MT: Diacetylmorphine versus methadone for the treatment of opioid addiction. N Engl J Med 2009;361:777-786.

10 Haasen C, Verthein U, Degkwitz P, Berger J, Krausz M, Naber D: Heroin-assisted treatment for opioid dependence: randomised controlled trial. Br J Psychiatry 2007;191:5562 .
11 van den Brink W, Hendriks V, Blanken P, Koeter M, van Zwieten B, van Rhee JM: Medical prescription of heroin to treatment resistant heroin addicts: two randomised controlled trials. BMJ 2003;327:310.

12 Kastelic A, Dubajic G, Strbad E: Slow-release oral morphine for maintenance treatment of opioid addicts intolerant to methadone or with inadequate withdrawal suppression. Addiction 2008;103:1837-1846.

13 Frick U, Gerlich M, Ammann J: Applikationsformen von Diacetylmorphin und unerwünschte Arzneimittelwirkungen: April 2001 bis Oktober 2003: Forschungsbericht aus dem Institut für Suchtforschung. Zürich, Institut für Suchtforschung, 2003.

14 Girardin F, Rentsch K, Schwab M, Maggiorini M, Pauli-Magnus C, Kullak-Ublick G Meier P, Fattinger K: Pharmacokinetics of high doses of intramuscular and oral heroin in narcotic addicts. Clin Pharmacol Ther 2003;74:341-352.

15 Perger L, Rentsch KM, Kullak-Ublick GA, Verotta D, Fattinger K: Oral heroin in opioid-dependent patients: pharmacokinetic comparison of immediate and extended release tablets. Eur J Pharm Sci 2009;36:421432.

16 Frick U, Rehm J, Kovacic S, Ammann J, Uchtenhagen A: A prospective cohort study on orally administered heroin substitution for severely addicted opioid users. Addiction 2006;101:1631-1639.

17 Gyr E, Bourquin D, Lehmann T, Hug I, Brenneisen R: Pharmacokinetics and pharmacodynamics of orally administered diacetylmorphine hydrochloride. J Anal Toxicol 1997;21:93.

18 Halbsguth U, Rentsch KM, Eich-Höchli D, Diterich I, Fattinger K: Oral diacetylmorphine (heroin) yields greater morphine bioavailability than oral morphine: bioavailability related to dosage and prior opioid exposure. Br J Clin Pharmacol 2008;66:781791.
19 Bundesamt für Gesundheit B: Handbuch heroingestützte Behandlung. Richtlinien, Empfehlung, Information. Bern, Bundesamt für Gesundheit, 2000.

20 Frick U, Ammann J, Kovacic S, Bürki C, Mohler M, Käser R, Gerlich M, Güttinger F, Gschwend P, Rehm J: Qualitätssicherung in der Heroin gestützten Behandlung (HegeBe) der Schweiz seit 2001: Methodische Anmerkungen zu Zielkriterien, Adjustierung des Patienten-Mix und Zentrumsvergleichen. Gesundheitsökonomie \& Qualitätsmanagement 2006;11:155-161.

21 Frick U, Güttinger F, Ammann J, Kovacic S, Rehm J, Uchtenhagen A: Kohortenstudie zur Erfassung der Sicherheit und Verträglichkeit von Diacetylmorphin in peroraler Verabreichung bei opioidabhängigen Probanden. Forschungsberichte aus dem Institut für Sucht- und Gesundheitsforschung. Zürich, Institut für Sucht- und Gesundheitsforschung, 2005

22 Fischer B, Rehm J, Brissette S, Brochu S, Bruneau J, El-Guebaly N, Noël L, Tyndall MW, Wild CT, Mun P, Baliunas D: Illicit opioid use in Canada - comparing social, health and drug use characteristics of untreated users in five cities (OPICAN study). J Urban Health 2005;82:250-266

23 Fischer B, Rehm J, Patra J, Firestone-Cruz M: Changes in illicit opioid use profiles across Canada. Can Med Assoc J 2006;75:13851387.

24 Frick U, Ammann J, Rehm J, Uchtenhagen A: Diacetylmorphin in peroraler Verabreichung bei opioid-abhängigen Probanden: 2-Jahres Follow-up: Forschungsbericht aus dem Institut für Sucht- und Gesundheitsforschung. Zurich, Research Institute for Public Health and Addiction, 2007.
Frick/Rehm/Zullino/Fernando/ Wiesbeck/Ammann/Uchtenhagen 